\title{
The Influence of Geometric Imperfections on the Non-linear Dynamical Behaviour of Parametrically Excited Rectangular Plates
}

\author{
M. J. Bugaru \\ Department of Engineering Sciences, University "Politehnica", Bucharest, Romania
}

\begin{abstract}
The author reviews recent developments in the non-linear forced and parametric vibrations of rectangular plates. The geometric imperfections of the plate are considered as a new parameter of the system and the problem is solved for one set of boundary-conditions: a simply-supported plate. The solution indicates the involvement of the geometric imperfections on the amplitude of forced vibrations and on the amplitude of parametric vibrations at the principal parametric resonance. In the mean time the interaction between forced vibrations and parametric vibrations is presented as a chaos behaviour. Numerical results reveal the influence of various system parameters and the experimental data verify the analytical solution from a qualitative and a quantitative point of view.
\end{abstract}

\section{NOMENCLATURE}

$A_{1 m}, A_{2 m}, A_{3 m}, B_{1 m}, B_{2 m}$ - unknown functions in asymptotic expansion

$C_{q}$ - viscous damping coefficient associated with a particular spatial mode of vibration

$D$ - flexural rigidity of plate

$E$ - Young's modulus

$F(X, Y, T)$ - dimensionless stress function

$F_{m n}(T)$ - time-dependent load factors in the assumed dimensionless stress function series

$H_{i j l}^{m}, M_{i j k}^{m}$ - coefficients of the non-linear terms

$N_{Y}(T)$ - dimensionless external in-plane loading per unit width

$N_{Y O}$ - dimensionless static in-plane loading per unit width

$N_{Y T}$ - dimensionless amplitude of harmonic in-plane loading per unit width

$N_{y}(t)$ - external in-plane loading per unit width

$N_{y o}$ - static in-plane loading per unit width

$N_{y t}$ - amplitude of harmonic in-plane loading per unit width

$N_{u v}$ - critical load corresponding to the $(u, v)$ buckling mode of the plate, defined as in reference ${ }^{14} \mathrm{pp} .353$

$R=b / a$ - plate aspect ratio

$T$ - dimensionless time

$W(X, Y, T)$ - dimensionless lateral midsurface displacement in Z-direction

$W_{0}(X, Y)$ - dimensionless initial geometric imperfection of plate in Z-direction

$W_{m f}(T)$ - dimensionless time-dependent amplitude of the forced vibration

$W_{m p}(T)$ - dimensionless time-dependent amplitude of the parametric vibration

$W_{p q}(T)$ - dimensionless time-dependent unknown function in the assumed dimensionless lateral displacement series

$X, Y, Z$-dimensionless space co-ordinates

$X_{m}(X), Y_{n}(Y)$ - spatial forms in the assumed stress function series (beam eigen-functions)

$a$ - length of plate in x-direction

$b$ - length of plate in y-direction $b_{m}$ - unknown non-linear function in asymptotic expansion

$d_{k l}$ - static deflection associated with a particular spatial mode of buckling

$f(x, y, t)$ - Airy's stress function

$h$ - plate thickness

$p, q$ - number of half-waves (in $\mathrm{X}$ and $\mathrm{Y}$-directions) in which the plate vibrates

$s=\Lambda / 2 \Omega_{m}$ - frequency parameter

$t$ - time

$w(x, y, t)$ - lateral midsurface displacement in z-direction

$w_{0}(x, y)$ - initial geometric imperfection in z-direction

$\Delta$ - decrement of damping

$\Theta(T)$ - dimensionless total phase angle of harmonic excitation

$\Lambda(T)$ - dimensionless instantaneous frequency of the external in-plane excitation, $\Lambda=d \Theta / d T$

$\Phi_{p}(X), \Psi_{q}(Y)$ - spatial forms in the assumed solution series for $W(X, Y, T)$

$\Omega_{q}$ - dimensionless free vibration circular frequency of a rectangular plate loaded by a constant component of in-plane force

$\bar{\Omega}_{q}$ - dimensionless free vibration circular frequency of a rectangular plate, with initial geometric imperfections, loaded by a constant component of in-plane force

$a_{m}, a_{n}$ - frequency parameters for beam eigen-functions $X_{m}, X_{n}$

$a_{i}$ - characteristic roots of the transcendental frequency equation

$\varepsilon$ - small positive parameter in asymptotic expansion, $0<\varepsilon<<1$

$\theta(t)$ - total phase angle of harmonic excitation

$\mu, \mu_{u v}$ - load parameter corresponding to the $(u, v)$ buckling mode of the plate

$v$ - Poisson's ratio

$\rho$ - mass density per unit volume of plate

$\tau$ - slowing time in asymptotic analysis

$\varphi_{m n}$ - static load factors in the assumed dimensionless stress function series

$\psi_{m f}(T)$ - phase angle of the forced vibration

$\psi_{m p}(T)$ - phase angle of the parametric vibration 